\title{
La colonisation grecque du bosphore Cimmérien
}

\section{Jurij Alekseevič Vinogradov}

Traducteur : Mathilde Reichler Imperiali

\section{OpenEdition \\ Journals}

Édition électronique

URL : http://journals.openedition.org/edl/324

DOI : $10.4000 /$ edl.324

ISSN : 2296-5084

\section{Éditeur}

Université de Lausanne

\section{Édition imprimée}

Date de publication : 15 mai 2012

Pagination : $57-86$

ISBN : 978-2-940331-27-7

ISSN : 0014-2026

\section{Référence électronique}

Jurij Alekseevič Vinogradov, «La colonisation grecque du bosphore Cimmérien », Études de lettres [En ligne], 1-2 | 2012, mis en ligne le 15 mai 2015, consulté le 19 décembre 2020. URL : http:// journals.openedition.org/edl/324 ; DOI : https://doi.org/10.4000/edl.324 


\section{LA COLONISATION GRECQUE DU BOSPHORE CIMMÉRIEN}

Les colons arrivés sur les rives du détroit de Kertch se sont trouvés en contact étroit non seulement avec les populations d'agriculteurs barbares (les Sindes et les Méotes), mais aussi avec des populations nomades (les Scythes). La colonisation des rives du détroit a commencé entre la fin du VII ${ }^{\mathrm{e}}$ s. et le début du VI ${ }^{\mathrm{e}}$ s. av. J.-C. Les mouvements périodiques des Scythes à travers le Bosphore (Hérodote IV.28), durant l'hiver, ont exercé une forte influence sur ce processus. Les Grecs ont fondé sur les rives du détroit une trentaine d'établissements, mais tous n'étaient pas des cités-Etats. Le recoupement des sources que nous possédons permet de supposer que sept de ces établissements étaient des poleis: Théodosie, Panticapée, Nymphaion, Phanagorie, Hermonassa, Kèpoi et Port des Sindes/Gorgippia, alors que les autres établissements furent probablement fondés à la suite de la colonisation interne de la région (Kytée, Myrmèkion, Tiritaka, Porthmion, etc.).

La colonisation grecque du nord de la mer Noire, dont la colonisation du Bosphore Cimmérien constitue une partie intégrante, représente un problème scientifique extrêmement complexe qui a donné lieu à une importante littérature vulgarisée et spécialisée ${ }^{1}$. Et pourtant, bien

I. Voir E. H. Minns, Scythians and Greeks, p. 563-566 et 569 sq., ainsi que M. I. Rostovtzeff, Iranians and Greeks in South Russia, p. 65 sq.; V. F. Gajdukevič, Das Bosporanische Reich, p. 15-31; Ju. G. Vinogradov, Pontische Studien, p. 5; G. A. Koshelenko, V. D. Kuznetsov, "Greek Colonisation of the Bosporus», p. 249263; A. A. Iessen, Grečeskaja kolonizacija Severnogo Pričernomor' ja, ejo predposylki i osobennosti; V. D. Blavatskij, "Arkhaičeskij Bospor»; V. V. Lapin, Grečeskaja kolonizacija Severnogo Pričernomor’ja; F. V. Šelov-Kovedjaev, «Istorija Bospora v VI-IV vv. do n. è.», p. 57-59; Ju. A. Vinogradov, "Nekotorye diskussionnye problemy grečeskoj kolonizacii Bospora Kimmerijskogo». 
des questions sur l'appropriation par les Grecs des rives du détroit de Kertch (l'ancien Bosphore Cimmérien) restent aujourd'hui encore très discutées. De plus, il est évident que toutes sortes de circonstances ont influencé le développement de la colonisation grecque, liées aussi bien au contexte dans la métropole qu'à celui prévalant sur la rive nord du Pont. Etant donné les dimensions restreintes de cet article, je n'aborderai pas des aspects aussi importants que la situation militaire et politique de l'Asie Mineure des VII et VI ${ }^{\mathrm{e}}$ s. av. J.-C., le développement de l'art de la navigation en Grèce, les connaissances grecques en matière de géographie, etc., qui ont tous, d'une manière ou d'une autre, agi sur le développement du processus de colonisation. Je ne voudrais pas non plus m'attarder sur un problème qui a longuement été débattu parmi les chercheurs russes et en particulier soviétiques, à savoir le but de la pénétration grecque dans cette région: les raisons en étaient-elles agricoles ou commerciales ? ${ }^{2}$ Une telle approche paraît aujourd'hui peu productive, et je ne voudrais pas perpétuer inutilement une dispute qui n'a que trop duré. Le but de mon article est de présenter de nouveaux matériaux archéologiques, découverts ces dernières années dans la région du détroit de Kertch, et de proposer quelques nouvelles approches dans l'étude de la colonisation grecque et dans l'étude de l'histoire première des colonies (apoikiai) grecques du Bosphore.

Le détroit de Kertch occupe la partie nord-est de la mer Noire: il baigne, à l'est, la péninsule de Taman, et à l'ouest, la Crimée orientale (péninsule de Kertch) (pl. II). Selon l'idée que s'en faisaient les géographes de la Grèce antique, c'est là que passait la frontière entre l'Europe et l'Asie (Hérodote IV.45; Strabon VII.4.5, XI.2.1); en conséquence, la péninsule de Taman faisait partie de l'Asie (Bosphore asiatique), tandis que l'est de la Crimée faisait partie de l'Europe (Bosphore européen). Avant toute chose, il est essentiel de relever que le Bosphore Cimmérien a été l'un des centres les plus importants de la colonisation grecque du nord de la mer Noire avec Olbia et Chersonèse Taurique. Mais on peut dire d'emblée bien plus: le Bosphore a été une sorte de pont entre deux parties du monde, entre différents peuples et différentes cultures locales. Il n'en demeure pas moins que malgré une situation géographique indubitablement avantageuse, nous ne savons presque rien de la population

2. Voir I. B. Brašinskij, A. N. Ščeglov, «Nekotorye problemy grečeskoj kolonizacii», p. 30 sq. 
du Bosphore avant l'arrivée des Grecs. On peut supposer qu'avant le début de la colonisation grecque, il ne devait exister sur les rives du détroit que de petits villages isolés et peu étendus.

Il est clair que la situation géographique du Bosphore représentait un atout très important pour les colons grecs. Les terres fertiles de part et d'autre du détroit promettaient d'abondantes récoltes de blé, les eaux regorgeaient de beaux poissons, etc. ${ }^{3}$. Pourtant A. Graham, il y a déjà quarante ans, remarquait à juste titre que les «geographical factors do not in themselves determine the pattern of Greek colonization " ${ }^{4}$. Les modes d'interaction des colons avec les populations locales ont constitué un facteur très important de la pénétration grecque dans les régions colonisées; plus précisément, leur niveau de développement économique et social a influencé le processus de colonisation.

En ce qui concerne le détroit de Kertch, les Grecs y ont été en contact étroit non seulement avec la population d'agriculteurs barbares du côté asiatique (des Sindes et des Méotes), mais aussi avec la population de nomades qui, au moment de la colonisation, était constituée de Scythes nomades. Il faut remarquer également que parmi les Etats grecs de la région, le Bosphore était le centre le plus oriental: il se trouvait effectivement sur le chemin des migrations de nomades d'est en ouest. Les mouvements périodiques des nomades vers l'ouest, qui avaient lieu tous les deux cents ans, ont été la cause de changements majeurs dans la situation militaire et politique des steppes, provoquant des répercussions sur le développement de tous les peuples et de tous les Etats qui touchaient cette zone. Ces rythmes de l'Eurasie ont joué un rôle très important pour l'histoire de la région du nord de la mer Noire, dans l'Antiquité comme au Moyen Age, déterminant les étapes historiques principales du développement de tout ce territoire 5 .

La colonisation du Bosphore, comme on le sait, a commencé entre la fin du VII ${ }^{\mathrm{e}}$ s. et le début du VI ${ }^{\mathrm{e}}$ s. av. J.-C., c'est-à-dire après celle du nord-ouest de la mer Noire (établissement de l'île de Bérézan). Il est assez étonnant par ailleurs que la pénétration grecque dans la région de la mer

3. Voir F. V. Šelov-Kovedjaev, «Istorija Bospora v VI-IV vv. do n. è.», p. 54 sq.

4. A. J. Graham, «Patterns in Early Greek Colonisation», p. 47.

5. K. K. Marčenko, Yu. A. Vinogradov, "The Scythian Period in the Northern Black Sea Region (750-250 BC)», p. 805-811; Ju. A. Vinogradov, "Cimmerian Bosporus", "Rhythms of Eurasia and the Main Historical Stages of the Kimmerian Bosporos in Pre-Roman Times» et "The Cimmerian Bosporus». 
d'Azov (le Méotide ancien) ait également commencé auparavant. Sur ce point, les vestiges de l'établissement antique situé près de la ville actuelle de Taganrog ont une importance capitale; il est possible qu'il s'agisse du port de Krèmnoi, mentionné par les auteurs anciens (Hérodote IV.20 et 110; Ptolémée III.5.4). Bien que l'établissement ait été recouvert par la mer d'Azov, on trouve souvent de la céramique grecque sur les plages de Taganrog. Ces découvertes permettent de supposer qu'un établissement grec fut fondé à cet endroit dans le troisième quart du VII ${ }^{\mathrm{e}} \mathrm{s}$. av. J.-C. ${ }^{6}$. Ainsi, la colonie de Taganrog apparaît comme l'un des établissements grecs les plus anciens de toute la côte nord de la mer Noire. Au point de vue chronologique, on ne peut la comparer qu'avec la colonie de l'île de Bérézan. Le mobilier archéologique provenant de ce site donne des informations extrêmement précieuses sur les débuts de la pénétration des Grecs dans la partie nord-est du Pont. De plus, il permet de comprendre l'une des particularités fondamentales de l'appropriation de territoires nouveaux par les colons. Cette appropriation ne s'est pas du tout déroulée progressivement!

Cette circonstance fait naître toute une série de questions qui nécessitent une explication scientifique. Tout d'abord, pourquoi les colons, ayant traversé le détroit de Kertch, n’ont-ils fondé aucune colonie sur ses rives? Pourquoi ce territoire est-il apparu aux Grecs comme «interdit", en quelque sorte? Pourquoi la colonie grecque la plus ancienne du nordest du Pont est-elle aussi la plus éloignée? Cette situation s'explique peutêtre par la situation démographique dans la région du détroit, et plus particulièrement par les migrations périodiques des Scythes à travers le Bosphore 7 . Hérodote a décrit ces migrations hivernales au livre IV de ses Histoires (IV.28). L'hypothèse la plus vraisemblable est que les Scythes ont traversé le détroit gelé dans sa partie la plus étroite, près de l'ancien Porthmion, où se trouve maintenant encore le passage de la Crimée vers $\operatorname{Taman}^{8}$. A cet endroit, la largeur du détroit est d'environ quatre kilomètres.

6. V. P. Kopylov, P. A. Larjonok, Taganrogskoe poselenie, p. 5; V. P. Kopylov, «Taganrogskoe poselenie v sisteme rannegrečeskikh kolonij Severnogo Pričernomor’ja».

7. M. Ju. Vakhtina, Ju. A. Vinogradov, E. Ja. Rogov, «Ob odnom iz maršrutov voennykh pokhodov i sezonnykh migracij kočevykh Skifov»; Ju. A. Vinogradov, "Bospor Kimmerijskij"; mais comparer avec Ju. G. Vinogradov, Pontische Studien, p. 109 sq.

8. Voir M. Ju. Vakhtina, «Porfmij - grečeskij gorod u perepravy čerez Kimmerijskij Bospor». 
Il ne fait aucun doute que les déplacements des nomades ont paru aux Grecs inhabituels et même dangereux. Il est possible qu'au vu de cette situation, ces derniers aient décidé d'éviter le détroit et de fonder leur colonie relativement près de celui-ci, sur la côte de la mer d'Azov. Depuis là, c'est-à-dire depuis un poste moins dangereux, ils avaient la possibilité de surveiller la situation du Bosphore. Ainsi, l'influence barbare sur le développement de la colonisation grecque dans cette région a commencé à se faire sentir dès le tout début du processus.

Le kourgane scythe de Temir-Gora, qui se trouve à l'est de la Crimée, non loin de la ville de Kertch, est un vestige archéologique important témoignant des migrations de nomades à travers le Bosphore. En 1870, au moment des fouilles de ce tertre, on découvrit une sépulture très représentative ${ }^{9}$. Elle consistait en un ensemble d'objets en os, ornementés dans ce qu'on appelle le style animalier des Scythes. On a aussi trouvé à cet endroit un vase grec peint, datant des années $40 \mathrm{du} \mathrm{VII}^{\mathrm{e}} \mathrm{s}$. av. J.-C. ${ }^{10}$. Ce vase apparaît comme l'objet le plus ancien provenant des kourganes scythes du nord de la mer Noire.

Pourtant, par la suite, entre le premier et le deuxième quart du $\mathrm{VI}^{\mathrm{e}} \mathrm{s}$. av. J.-C., malgré les traversées répétées des nomades en hiver, toute une série de colonies grecques furent fondées sur les rives du détroit. Les auteurs anciens nomment environ trente colonies de ce genre; certaines d'entre elles sont qualifiées de villes, d'autres de petites villes ou même de villages ${ }^{11}$.

Je ne peux être d'accord avec la conception de la colonisation grecque qui traite toutes les colonies grecques du début comme des apoikiai ou des city-states. Le problème de la polis grecque coloniale, comme on le sait, est extrêmement complexe et délicat pour l'analyse scientifique. La formule "une ville - une apoikia - une polis» me paraît trop générale. Bien sûr, il existait dans le Bosphore des établissements importants, fondés dès le début comme des apoikiai ou des city-states; mais en ellemême cette appellation nécessite des preuves, une confirmation à travers des matériaux concrets. Dans un article consacré à cette question, j’ai

9. M. I. Artamonov, Sokrovišča skifskikh kurganov v sobranii Gosudarstvennogo Ermitaža, p. 25 ; L. K. Galanina, N. L. Grach, Scythian Art, p. 20 sq.

Io. L. V. Kopejkina, «Raspisnaja rodossko-ionijskaja ojnokhoja iz kurgana TemirGora».

II. Voir V. F. Gajdukevič, Das Bosporanische Reich, p. 32-38 et 170-255. 
essayé de proposer une possibilité de séparation des poleis du Bosphore, en m'appuyant sur trois catégories de sources: les informations concernant la fondation des colonies, les ethnika des habitants et la monnaie ${ }^{12}$. Les sources écrites sur la colonisation grecque du Bosphore sont tout à fait restreintes, mais nous avons tout de même des témoignages concernant la fondation de six colonies: Théodosie, Panticapée et Kèpoi ont été fondées par des Grecs de Milet, Phanagorie par des Grecs de Téos, Hermonassa par des Ioniens ou des Eoliens, Gorgippia (à l'origine Port des Sindes) par des Grecs du Bosphore. Il est étonnant que les auteurs anciens, ayant décrit les événements de la plus lointaine histoire du Bosphore, n'aient mentionné en fait que ces colonies et n'aient pas dit un mot de Myrmèkion, Tiritaka ou Porthmion.

Ces témoignages, dans l'ensemble, sont confirmés par les données sur les ethnika et sur la monnaie du Bosphore. Dans les sources écrites et dans les documents épigraphiques, on trouve les ethnika des habitants (citoyens?) de Théodosie, Panticapée, Nymphaion, Phanagorie, Kèpoi, Hermonassa et Gorgippia. Le monnayage apparait bien entendu comme une preuve importante du statut de polis de la ville grecque. Les villes bosporanes à battre monnaie aux $\mathrm{VI}^{\mathrm{e}}$ et $\mathrm{V}^{\mathrm{e}} \mathrm{s}$. av. J.-C. étaient Panticapée, Théodosie, Nymphaion, Phanagorie, et plus tard Gorgippia ${ }^{13}$. Toutes ces données sont reproduites dans le tableau ci-contre.

On observe aisément, grâce à ce tableau, que tous les groupes de sources ne coïncident pas. Néanmoins, il y a matière à supposer que Théodosie, Panticapée, Nymphaion, Phanagorie, Hermonassa, Kèpoi et Gorgippia étaient de véritables poleis, tandis que les autres établissements - quelques douzaines, mentionnés dans les sources écrites et dans les données archéologiques - furent probablement fondés à la suite de la colonisation interne de la région (Kytée, Myrmèkion, Tiritaka, Porthmion, etc.); de ce fait, ils devaient vraisemblablement faire partie de telle ou telle polis ${ }^{14}$. Bien sûr, je ne peux pas exclure totalement le fait que certaines de ces colonies relativement petites n'aient été fondées comme des poleis, mais ce serait une exception qui ne changerait pas la règle elle-même.

I2. Ju. A. Vinogradov, «K probleme polisov v rajone Bospora Kimmerijskogo», ainsi que "Bospor Kimmerijskij", p. 221-223.

13. Voir D. B. Šelov, Coinage of the Bosporus VI-II centuries BC.

I4. Comparer avec G. R. Tsetskhladze, "A Survey of the Major Urban Settlements in the Cimmerian Bosporos». 


\begin{tabular}{|l|c|c|c|}
\hline Villes & $\begin{array}{c}\text { Information concer- } \\
\text { nant la fondation }\end{array}$ & Ethnikon & Monnaie \\
\hline Théodosie & + & + & + \\
\hline Panticapée & + & + & + \\
\hline Phanagorie & + & + & + \\
\hline Hermonassa & + & + & - \\
\hline Kèpoi & + & + & - \\
\hline Nymphaion & - & + & + \\
\hline $\begin{array}{l}\text { Port des Sindes/ } \\
\text { Gorgippia }\end{array}$ & + & - & - \\
\hline Myrmèkion & - & - & - \\
\hline Tiritaka & - & + & - \\
\hline Porthmion, etc. & + & + \\
\hline Tanaïs & & + & + \\
\hline
\end{tabular}

Villes du Bosphore Cimmérien.

(les cités-Etats sont désignées en italique.)

On peut comparer le résultat obtenu avec les données relativement tardives connues sur Tanaïs. Cette colonie grecque fut fondée sur le delta $\mathrm{du}$ Don dans le premier quart du III ${ }^{\mathrm{e}}$ s. av. J.-C. ${ }^{15}$. Strabon rapporte que Tanaïs fut fondée par des habitants du Bosphore (Strabon XI.2.3) et qu'elle joua un rôle très important pour l'histoire régionale ${ }^{16}$. Tanaïs n'a jamais frappé sa propre monnaie, mais on connaît l'ethnikon d'une partie de sa communauté de citoyens: les Tanaïtes ${ }^{17}$. A nouveau, malgré l'absence de certains signes importants du statut de polis, on peut supposer avec certitude que Tanaïs apparaissait comme une véritable polis autonome ${ }^{18}$. A ce propos, il est impératif de souligner encore une fois que pour une compréhension adéquate de la colonisation grecque du

I5. D. B. Šelov, Tanais i Nižnij Don v III-I vv. do n.è., p. 23; T. M. Arsenyeva, «Tanais», p. 1052.

16. Voir D. B. Šelov, Tanais i Nižnij Don v III-I vv. do n.è.

17. V. F. Gajdukevič, Das Bosporanische Reich, p. 251, 254 sq., 337, 362 sq.; T. M. Arsenyeva, «Tanais», p. 1081.

I8. D. B. Šelov, Tanais i Nižnij Don v III-I vv. do n.è., p. 201 sq. et "Tanais èllinističeskij gorod», p. 48 sq. 
Bosphore, il est fondamental de mettre en exergue les colonies grecques les plus importantes de cette région, et non pas de les assimiler les unes aux autres en se référant aux conceptions vagues de petites poleis, petites villes, etc.

Il est extrêmement curieux que les établissements grecs les plus anciens du Bosphore européen aient été de dimensions relativement importantes et que plus tard, ils aient été de surcroît transformés en villes. On peut supposer que cette situation s'explique à nouveau par des facteurs démographiques: la proximité de la Scythie du Caucase septentrional ${ }^{19}$ et les mouvements périodiques des groupes de Scythes à travers le détroit gelé (déplacements mentionnés par Hérodote IV.28). Les vestiges archéologiques permettent de penser que les nomades avaient fondé une union très puissante dans la région nord du Caucase. Les chefs de cette union, de toute évidence, pouvaient sans difficulté exercer leur contrôle sur la région du détroit, et même exiger des Grecs certains tributs, des cadeaux coûteux, etc. Pourtant le facteur le plus important à intervenir dans le bilan démographique du Bosphore, comme je l'ai déjà dit, tient dans les déplacements périodiques des Scythes. Il n'y a aucun doute que ceux-ci représentaient un danger non négligeable pour les Grecs. Le plus plausible est que ce soit justement pour cette raison que les colons bosporans n'aient pas pu créer un système de colonies agricoles dans les environs de leurs villes, comparable à la chôra archaïque d'Olbia ${ }^{20}$. La construction de villages sur le chemin des nomades était une entreprise vouée à l'échec. Cette situation a apparemment contraint les Grecs du Bosphore à s'installer dans des colonies relativement importantes et pas trop éloignées les unes des autres. Par la suite, certaines d'entre elles se sont transformées en "petites villes agraires", incluses dans le territoire rural de poleis plus importantes telles Panticapée, Nymphaion ou Phanagorie.

Malgré cette situation démographique spécifique, il existe de sérieuses raisons de penser que les établissements coloniaux grecs ont d'abord vécu dans une atmosphère paisible, sans signe manifeste de menace extérieure, d'attaque guerrière, etc. Il est probable que lors de la première étape de la

19. Voir A. M. Khazanov, Social'naja istorija Skifov, p. 226; A. Ju. Alekseev, Khronografija evropejskoj Skifii VII-IV vv. do n.è., p. 103 sq.; V. Yu. Murzin, S. A. Skory, "An Essay of Scythian History», p. 57.

2o. Voir S. D. Kryžitckij, S. B. Bujskikh, A. V. Burakov, V. M. Otreško, Sel'skaja okruga Ol'vii, p. 22 sq.; S. D. Kryžitckij, S. B. Bujskikh, V. M. Otreško, Antičnye poselenija Nižnego Pobuž'ja, p. 10 sq. 
colonisation, les Grecs aient su créer de bonnes relations avec les populations barbares locales, que ce soient les Méotes ou les Scythes. En ce qui concerne Panticapée, nous avons même l'information qu'elle fut fondée

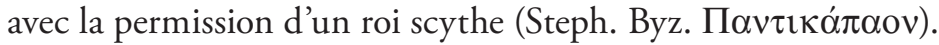

Pourtant, vers la moitié du $\mathrm{VI}^{\mathrm{e}}$ s. av. J.-C., les colons grecs rencontrèrent de grosses difficultés. Ces difficultés s'expriment en premier lieu par des incendies de grande envergure, dont les archéologues ont découvert la trace à Kèpoi, Myrmèkion et Porthmion. A la même époque, l'établissement de Taganrog, sur la côte de la mer d'Azov, a tout simplement cessé d'exister ${ }^{21}$. Il est révélateur qu'on ait découvert assez récemment à Myrmèkion et à Porthmion les vestiges d'une ancienne fortification datant approximativement de la moitié du VIe s. av. J.-C. ${ }^{22}$. Celle-ci apparaît comme l'exemple de système de fortification le plus ancien qui soit connu aujourd'hui dans les colonies grecques du nord de la mer Noire. Il semble que tous ces événements (incendies et construction d'édifices défensifs) soient liés aux attaques des nomades, bien que les attaques barbares de cette époque n'aient pas provoqué de situation de crise dans les steppes du nord de la mer Noire. Il se peut qu'elles n'aient été que les prémices des bouleversements à venir.

Le caractère unique de la fortification ancienne de Myrmèkion tient au fait que seule l'acropole a été fortifiée, et non toute la superficie de la colonie $^{23}$. Les murs de fortification ont été dégagés sur une longueur totale de vingt mètres. Leur fondation, faite de grandes pierres calcaires grossièrement taillées, mesure plus d'un mètre de largeur. Les interstices entre ces grosses pierres ont été comblés avec de petits cailloux. Les murs eux-mêmes devaient probablement être faits de briques en terre crue. Les ouvrages de fortification qui défendaient l'acropole démontrent avec évidence l'habileté des constructeurs, qui ont utilisé avec succès la topographie de l'endroit (pl. III.1). Les murs fermaient avant tout les zones qui permettaient de monter au sommet. C'est le principe du bastion qui a été

2I. V. P. Kopylov, "Taganrogskoe poselenie v sisteme rannegrečeskikh kolonij Severnogo Pričernomor'ja», p. 174 sq.

22. M. Ju. Vakhtina, Ju. A. Vinogradov, «Eščje raz o rannej fortifikacii Bospora Kimmerijskogo".

23. Ju. A. Vinogradov, «Selecting Findings from the Myrmekion Acropolis», p. 290292; Ju. A. Vinogradov, A. M. Butyagin, M. Yu. Vakhtina, "Myrmekion - Porthmeus», p. 806 sq.; Ju. A. Vinogradov, «Nekotorye itogi arkheologičeskikh issledovanij v rajone akropolja Mirmekija» et «Die Befestung der Akropolis von Myrmekion». 
utilisé pour la construction de ce système défensif, un type de disposition qui ne nécessitait pas d'ériger de tours. De manière générale, on doit reconnaître que le système de fortification de l'acropole de Myrmèkion est parfaitement réussi. D’un autre côté, il faut avouer que ce système est apparu en même temps que les cabanes semi-enterrées ${ }^{24}$ et relativement primitives qui constituaient Myrmèkion, donnant à la colonie l'air d'un amoncellement désordonné de constructions d'aspect «semi-barbare».

En ce qui concerne l'étude archéologique de la Myrmèkion archaïque, nous avons encore d'autres découvertes importantes à faire valoir. Les fouilles de l'établissement menées dans les années 80 et 90 du siècle passé se sont concentrées sur l'étude des couches les plus anciennes. Ainsi, au pied du rocher de ce qu'on appelle l'acropole de Myrmèkion, on a découvert toute une série de fosses datant du deuxième quart du $\mathrm{VI}^{\mathrm{e}} \mathrm{s}$. av. J.-C. ${ }^{25}$. De telles découvertes sont extrêmement rares sur les sites archéologiques du Bosphore. Parmi les trouvailles, on peut mentionner des fragments de céramique corinthienne peinte, d'amphores décorées dans le style de la chèvre sauvage (Wild Goat Style), etc. (pl. III.2-3 et IV.1-2). Toutes ces découvertes permettent de penser que Myrmèkion a été fondée durant le deuxième quart du $\mathrm{VI}^{\mathrm{e}} \mathrm{s}$. av. J.-C., et peut-être même au tournant du premier et du deuxième quart de ce siècle.

Il est essentiel de rappeler encore une fois que les résultats de la recherche archéologique contemporaine sur les villes du Bosphore permettent de penser qu'à l'origine, pratiquement toutes ces villes devaient avoir une allure "semi-barbare", car elles étaient constituées de cabanes semi-enterrées de dimensions modestes et très simples par leur construction ${ }^{26}$ (pl. IV.3). Certains chercheurs voient dans ces constructions l'ouvrage d'une population barbare, tandis que d'autres rejettent cette hypothèse, estimant que toutes ces constructions enfouies dans le sol et découvertes dans les colonies grecques, "cannot be taken to

24. Il est question de ce que l'on nomme dug out dans la littérature scientifique anglo-saxonne (ndlt).

25. Ju. A. Vinogradov, "Selecting Findings from the Myrmekion Acropolis»; Ju. A. Vinogradov, A. M. Butyagin, M. Yu. Vakhtina, «Myrmekion - Porthmeus», p. 805 sq.; Ju. A. Vinogradov, «Nekotorye itogi arkheologičeskikh issledovanij v rajone akropolja Mirmekija», p. 44-48.

26. G. R. Tsetskhladze, "On the Earliest Greek Colonial Architecture in the Pontus», p. 225 sq. 
show ethnicity» ${ }^{27}$. V. D. Kuznecov rejette de manière générale l'existence même de ce type de construction dans les colonies grecques de la rive nord du Pont ${ }^{28}$, ce qui apparaît clairement comme une méprise. Toutefois, les deux conceptions évoquées ci-dessus sont elles aussi exagérées! Les colons ont très bien pu utiliser des méthodes de construction simples pour leurs habitations, caractéristiques des aborigènes, et le point de vue qui considère cette tradition de construction semi-enterrée dans les colonies grecques comme le signe d'une influence barbare me paraît plus réaliste. En même temps, il est indispensable de souligner que la structure et l'aspect extérieur des premières colonies grecques du Bosphore Cimmérien et, de manière générale, de tout le nord de la mer Noire, se distinguent des colonies que l'on connaît en Méditerranée occidentale. C'est là, comme on le sait, qu'on a pu mettre en évidence les plus anciens plans d'aménagement de villes grecques. De telles découvertes dans la région nord de la mer Noire, comme ce dont nous allons parler plus loin, doivent être considérées, à mon avis, non pas comme une règle, mais plutôt comme une exception.

La "phase de construction semi-enterrée» caractéristique de cette région s'achève environ soixante-dix ou quatre-vingts ans après la fondation de l'établissement, c'est-à-dire seulement du temps de la deuxième ou même de la troisième génération de colons ${ }^{29}$. La fin de cette étape est marquée par le fait que toutes les cabanes semi-enterrées sont comblées de terre; à leur place, on a élevé des maisons "en surface» avec des soubassements de pierre pour les parois et on a, en outre, tracé des rues, posé des dalles en pierre, etc. (pl. V). Ce tournant décisif est bien visible à Myrmèkion, où il se produit entre les $\mathrm{VI}^{\mathrm{e}}$ et $\mathrm{V}^{\mathrm{e}}$ s. av. J.-C. ${ }^{30}$. C'est seulement après ce réaménagement essentiel que les colonies grecques les plus importantes acquièrent une véritable structure urbanistique. Il faut traiter ce phénomène, de toute évidence, comme l'aboutissement d'une période d'adaptation des colons à des conditions plutôt difficiles au

27. S. D. Kryzhitskiy, "Criteria for the Presence of Barbarians in the Population of Early Olbia", p. 18 sq.

28. V. D. Kuznecov, «Kepoi-Phanagoria-Taganrog», p. 919 sq.

29. Ju. A. Vinogradov, «Grečeskaja kolonizacija i grečeskaja urbanizacija Severnogo Pričernomor'ja», p. 107 sq. et "K probleme stanovlenija drevnegrečeskikh gorodov v rajone Bospora Kimmerijskogo».

30. Ju. A. Vinogradov, A. M. Butyagin, M. Yu. Vakhtina, «Myrmekion Porthmeus", p. 809. 
niveau climatique, écologique et démographique. A partir de ce moment et environ jusqu'en 480 av. J.-C., les colonies du Bosphore Cimmérien ont été au sommet de leur épanouissement économique et culturel. La fin de la période d'adaptation est marquée par l'apparition de la monnaie bosporane. Panticapée a commencé à produire sa monnaie en argent probablement vers le dernier tiers du VIe s. av. J.-C., c'est-à-dire pratiquement en même temps que l'apparition, à cet endroit, de maisons en pierre ${ }^{31}$. Les datations plus anciennes de cet événement qui ont été formulées sont, en général, purement spéculatives ${ }^{32}$.

La présence d'artefacts typiques des cultures locales (avant tout de la céramique modelée, fabriquée sans tour de potier) est une caractéristique des stratifications anthropiques des établissements grecs du nord de la mer Noire et, en particulier, du Bosphore ${ }^{33}$. L'apparition d'une telle céramique, ici, est incontestablement liée à l'implantation de personnes issues de la population barbare. Je ne peux être d'accord avec les chercheurs (en premier lieu, avec mes collègues de Kiev), qui contestent ce point de vue et qui affirment que la céramique modelée, dans les communautés coloniales grecques, "cannot serve at all as reliable evidence on ethnicity» ${ }^{34}$.

Il est étonnant que la céramique modelée soit la plus nombreuse dans les couches anciennes des colonies grecques. A Myrmèkion, par exemple, elle constitue $24 \%$ à $37 \%$ de tout le complexe céramique ${ }^{35}$. Dans les stratifications plus tardives, ce pourcentage se réduit à moins de la moitié. De plus, les matériaux anciens des colonies du Bosphore peuvent être répartis en trois groupes importants, qui présentent des ressemblances avec la céramique des différents groupes de la population locale: la céramique de la steppe (scythe), de la Crimée des montagnes (taurique) et la céramique de la région du Kouban (méote). L'étude attentive de la céramique modelée des villes du Bosphore prouve encore un fait de toute première importance: à savoir que les différentes colonies grecques ont

3I. V. A. Anokhin, Istorija Bospora Kimmerijskogo, p. 6; A. E. Tereščenko, «O pojavlenii monetnogo dela na Bospore Kimmerijskom».

32. V. F. Gajdukevič, Das Bosporanische Reich, p. 523; D. B. Šelov, Coinage of the Bosporus VI-II centuries BC, p. 10 sq.

33. Voir E. G. Kastanajan, Lepnaja keramika bosporskikh gorodov.

34. S. B. Buyskikh, "Contacts between Greeks and Non-Greeks on the Lower Bug in the Sixth and Fifth Centuries BC», p. 32.

35. Ju. A. Vinogradov, «Mirmekij», p. 102. 
eu des contacts étroits surtout avec tel ou tel peuple barbare. Dans le mobilier archéologique de Nymphaion, par exemple, on peut observer des liens avec la culture de la population des montagnes de Crimée, alors que Myrmèkion entretenait des contacts avec les Méotes, c'est-à-dire avec des tribus qui étaient établies de l'autre côté du Bosphore.

A propos de ces observations de type archéologique, il faut relever que l'ethnographie, aujourd'hui, a démontré de manière convaincante que la production de céramique modelée était liée à l'activité des femmes. On peut donc supposer que les colons grecs étaient en contact avec les tribus locales non seulement au niveau économique et politique, mais aussi dans le cadre de relations conjugales. De tels liens garantissaient le maintien du potentiel démographique des communautés de colons grecs créées depuis peu ${ }^{36}$. On peut dire avec une quasi-certitude que la formation de poleis dans le Bosphore a dû conduire à des mariages mixtes entre Grecs et Barbares. Il convient de se ranger à l'opinion de D. Braund qui conclut, à propos de la colonie d'Olbia, que la plupart des colons, si ce n'est même tous les colons, étaient des hommes jeunes, surtout au stade premier du processus de colonisation, et c'est principalement en milieu barbare qu'ils pouvaient se trouver des fiancées ${ }^{37}$. Il devait y avoir dans le Bosphore, semble-t-il, une situation similaire. Dans la mesure où la céramique des colonies archaïques du Bosphore contient une quantité non négligeable de vaisselle indigène modelée, on peut supposer en effet que la présence barbare, en particulier la présence de femmes barbares, était ici tout à fait importante. Il est curieux, par ailleurs, qu'avec la création d'une structure urbanistique dans les colonies du Bosphore, comme le montre l'exemple de Myrmèkion, la quantité de céramique modelée dans les couches anthropiques diminue sensiblement, tandis que ses formes se simplifient ${ }^{38}$.

Ce fait nous persuade encore une fois de la pertinence de la conception selon laquelle il convient de parler du caractère grec des villes $\mathrm{du}$ Bosphore comme des autres centres de la colonisation grecque de cette région. La langue écrite était le grec, et les dieux grecs constituaient la base du système religieux avec, peut-être, quelques particularités

36. M. H. Hansen, "The Hellenic Polis», p. 143.

37. D. Braund, "Greater Olbia», p. 40.

38. Ju. A. Vinogradov, A. M. Butyagin, M. Yu. Vakhtina, «Myrmekion Porthmeus", p. 808 sq. 
locales. De manière générale, on peut conclure qu'au cours de l'époque archaïque, toutes les influences indigènes furent amalgamées dans une nouvelle unité, qui était grecque, ou en tout cas passablement grecque ${ }^{39}$.

Phanagorie est la colonie grecque du Bosphore la plus tardive. Nous savons que l'oikistès de Phanagorie était Phanagoros de Téos, qui «sought refuge from Persian interference" (Arrien, Bithynica fr. 55; ed. RoosWirth). Cette information succincte nous permet de supposer qu'une partie importante de la communauté de Téos avait trouvé refuge dans le Bosphore. De plus, elle donne une base pour proposer une date assez précise de la fondation de la colonie entre 550 et 540 av. J.-C., date qui concorde bien avec le matériel archéologique découvert au moment des fouilles de la ville ${ }^{40}$. L'un des résultats importants de l'étude archéologique de Phanagorie est la découverte d'un tracé de ville ancien qui, comme on l'a dit, n'est pas caractéristique des autres colonies grecques du Bosphore. Selon la conclusion d'un chercheur ayant participé aux fouilles, V. D. Kuznecov, le plan parcellaire a été ici posé, sinon au moment de la fondation de la colonie, tout du moins, sans aucun doute, à l'époque archaïque ${ }^{41}$. En outre, selon une déclaration dudit chercheur lors d'une conversation orale avec l'auteur de ces lignes, on peut considérer que la céramique modelée, fabriquée traditionnellement dans les tribus barbares, ne se rencontre quasiment pas dans les couches les plus anciennes du site de Phanagorie. Une telle situation est tout à fait exceptionnelle pour l'archéologie antique du nord de la mer Noire! Il faut peut-être en chercher l'explication dans le fait que Phanagorie a été fondée par un groupe d'immigrants de Téos, qui comptait un nombre certain de femmes, ce qui implique que les citoyens de la nouvelle colonie n'eurent pas à aller chercher leurs fiancées à l'extérieur.

Le processus de colonisation, comme on l'a déjà dit, est lié à la transformation de la culture des colons, mais aussi à la transformation du système économique. On sait que l'exploitation rurale, l'artisanat et le commerce constituaient la base de l'économie de la Grèce. En ce qui concerne l'économie du Bosphore, on peut relever que les villes importantes (Panticapée, Phanagorie, Nymphaion et les autres) furent des centres de production artisanale et de commerce, mais toutes étaient

39. D. Braund, "Greater Olbia», p. 44.

40. V. D. Kuznecov, «Kepoi-Phanagoria-Taganrog», p. 898 et 906.

4I. V. D. Kuznecov, «Fanagorija», p. 10. 
également liées de manière extrêmement étroite à l'exploitation rurale. D'autre part, n'oublions pas qu'à l'époque archaïque, les opérations de trocs avec les tribus locales étaient plutôt limitées. Il semble qu'elles concernaient en premier lieu la sphère des relations avec l'aristocratie barbare.

Certains axes de la transformation économique des colonies grecques du nord de la mer Noire paraissent aujourd'hui tout à fait évidents. En ce qui concerne l'élevage, il faut reconnaître l'influence significative des tribus autochtones, qui pouvaient fournir aux Grecs les espèces d'animaux les plus appropriées aux conditions locales ${ }^{42}$. A propos de l'agriculture de la Grèce antique, on sait qu'elle se basait sur ce qu'on appelle la "triade méditerranéenne" ou la "tétrade méditerranéenne " ${ }^{43}$. La triade est constituée de la culture de l'olive, du raisin et des céréales, et la tétrade comprend, en plus, les légumineuses. Il est clair qu'un tel assortiment ne pouvait pas être cultivé intégralement dans les conditions climatiques spécifiques du nord de la mer Noire, où les oliviers ne peuvent tout simplement pas pousser. En ce qui concerne le raisin, les colons grecs apprirent à le cultiver (Strabon VII.3.18), mais pas avant le III ${ }^{\mathrm{e}}$ s. av. J.-C. ${ }^{44}$, c'est-à-dire après trois cents ans de présence dans cette région.

Les colons ont transformé le modèle méditerranéen en une forme spécifique ${ }^{45}$, qu'on peut appeler le "duo du Pont nord». Les céréales (en particulier le blé tendre, Triticum aestivum s.l., et l'orge commune, Hordeum vulgare) formaient la première de ses composantes, et les légumineuses la deuxième. A ce propos, il est essentiel de préciser que trois ensembles très caractéristiques de graines brûlées issues de plantes de culture ont été découverts lors des fouilles à Myrmèkion ${ }^{46}$. Ils proviennent d'époques différentes: le premier est daté du deuxième quart du VIe s. av. J.-C., le deuxième du dernier quart de ce même siècle, et le troisième du premier quart du $\mathrm{V}^{\mathrm{e}}$ s. av. J.-C. Ils présentent presque toujours le même contenu:

42. Ju. A. Vinogradov, «Prirodnij faktor v razvitii Bospora v skifskuju èpokhu (Nekotorye aspekty izučenija)», p. 86-88.

43. A. Sarpaki, "The Palaeoethnobotanical Approach»; S. Isager, J. E. Skydsgaard, Ancient Greek Agriculture, p. 26 sq., 33 sq., 42 sq.

44. V. F. Gajdukevič, "Vinodelie na Bospore».

45. G. A. Pashkevich, «Archaeobotanical studies on the northern coast of the Black Sea", p. 530-535.

46. Ju. A. Vinogradov, "K izučeniju zeren kul'turnykh rastenij, najdennykh v Mirmekii». 
blé tendre, orge, légumineuses. La quantité de légumineuses, de plus, est relativement peu importante: elle est dix fois inférieure à celle des céréales. Le blé et l'orge sont représentés approximativement dans des proportions égales. Ainsi, on peut supposer que l'éventail des cultures agricoles, différent de celui de la métropole, s'est constitué très rapidement dans le Bosphore, au tout début de la colonisation grecque de cette région.

Il faut porter une attention particulière au blé, dans la mesure où il forme la part la plus importante du commerce céréalier du Bosphore avec la Méditerranée qui était si renommé au $\mathrm{IV}^{\mathrm{e}}$ et au début du III ${ }^{\mathrm{e}}$ s. av. J.-C. (Diodore XX.32; Strabon VII.4.6) ${ }^{47}$. L'orge faisait partie de la nourriture du commun de la population et, sur les marchés de la Méditerranée, il ne coûtait pas cher. Le blé, par contre, était passablement plus cher. Les colons pouvaient l'exporter avec succès dans la métropole, recevant en échange des sommes importantes. Cette exportation garantissait aux apoikiai du Bosphore d'être approvisionnées en vin, en huile d'olive et dans tous les autres produits indispensables et objets artisanaux qui n'étaient pas produits chez eux.

Nous avons déjà parlé, plus haut, des colonies rurales de l'époque archaïque dans le Bosphore Cimmérien. Il apparaît donc évident que l'étude archéologique des territoires agricoles ou des chôrai de chaque polis revêt une signification toute particulière pour la compréhension de son histoire et sur ce point, beaucoup a été fait sur le Bosphore ${ }^{48}$. Pourtant, l'étude comparative des chôrai des Etats grecs du nord de la mer Noire est également riche de perspectives. Si l'on compare les données archéologiques des territoires agricoles de l'époque archaïque d'Olbia avec celles du Bosphore, le résultat est tout à fait révélateur. Dans les environs d'Olbia, on connaît environ deux cents colonies agricoles permanentes ${ }^{49}$, alors qu'en Crimée orientale, on n'en connaît qu'une

47. V. F. Gajdukevič, Das Bosporanische Reich, p. 97 sq.; mais comparer avec V. D. Kuznecov, "Afiny i Bospor».

48. I. T. Kruglikova, Sel'skoe khozjajstvo Bospora; A. A. Maslennikov, Ellinskaja khora na kraju Ojkumeny; S. Ju. Saprykin, "The Chora in the Bosporan Kingdom", p. 273 sq.

49. S. D. Kryžitckij, S. B. Bujskikh, A. V. Burakov, V. M. Otreško, Sel'skaja okruga Ol'vii, p. 22 sq.; S. D. Kryžitckij, S. B. Bujskikh, V. M. Otreško, Antičnye poselenija Nižnego Pobužja, p. 10 sq. 
dizaine environ ${ }^{50}$. Ces exemples témoignent de systèmes différents d'implantation grecque: dans le Bosphore européen, les colons ne purent pas créer de système de chôra comme ils le faisaient habituellement, c'està-dire en construisant des villages. Une telle situation, comme je l'ai déjà mentionné plusieurs fois, peut s'expliquer par les déplacements des Scythes à travers le détroit gelé de Kertch.

Sur la péninsule de Taman, la situation n'est pas aussi claire; néanmoins il est indéniable que c'est la partie asiatique qui servit de véritable grenier du Bosphore tout au long de son histoire. Les recherches archéologiques menées à cet endroit prouvent l'existence de plus d'une centaine de colonies archaïques agricoles ${ }^{51}$. Certains chercheurs tentent d'expliquer cette situation «by the different roles that Scythian played in the two regions " ${ }^{2}$. Mais en fait, d'un point de vue concret, toutes les colonies agricoles de la péninsule de Taman nécessiteraient encore une investigation archéologique systématique.

A ce propos, on peut porter notre attention sur l'une des colonies agricoles bien étudiées de la région: Artjuščenko-1 (ou Bugazskoe) ${ }^{53}$. Elle se trouve dans la partie sud de la péninsule, sur la côte escarpée de la mer Noire. Cette colonie faisait probablement partie du territoire agricole d'Hermonassa, une polis grecque qui s'étendait sur les lieux actuels du village cosaque de Taman. A l'époque archaïque (dernier tiers du $\mathrm{VI}^{\mathrm{e}}$ s. - premier tiers du $\mathrm{V}^{\mathrm{e}}$ s. av. J.-C.), Artjuščenko-1 était une colonie saisonnière. Son mobilier archéologique est très pauvre: quelques petites cabanes semi-enterrées primitives, et une multitude de simples fosses. Parmi les objets de céramique trouvés dominent des fragments d'amphores grecques (principalement des amphores de Lesbos en argile rouge) et des débris de vaisselle modelée. On a trouvé également quelques fragments isolés de vaisselle grecque peinte. Il est essentiel de constater que dans ce complexe céramique, plus de $50 \%$ des trouvailles concernent

50. Ju. A. Vinogradov, «Bospor Kimmerijskij», p. 223-224; S. Ju. Saprykin, "The Chora in the Bosporan Kingdom», p. 273-275.

51. A. P. Abramov, Ja. M. Paromov, «Ranneantičnye poselenija Tamanskogo poluostrova", p. 71 sq.

52. G. A. Koshelenko, V. D. Kuznetsov, "Greek Colonisation of the Bosporus", p. 262.

53. Ju. A. Vinogradov, E. V. Lebedeva, «Excavations at the Classical-Period Settlement of Artyushchenko-1 (Bugazskyoe) on the Taman Peninsula». 
de la vaisselle modelée, fabriquée selon les traditions de la population agricole locale de la région du Kouban (des tribus méotes) ${ }^{54}$ (fig. 1 et 2).

La composition ethnique de pratiquement tous les établissements de la chôra grecque dans le nord de la mer Noire est tout à fait variable, et le pourcentage de Barbares était plus haut dans les colonies agricoles que dans les villes. Les nombreuses découvertes de céramique modelée présentant une ressemblance manifeste avec la céramique des tribus indigènes confirme cette donnée. Mais $50 \%$ de vaisselle modelée pour le complexe céramique recensé à Artjuščenko-1, c'est un chiffre trop élevé! Dès lors, on peut supposer que les agriculteurs de cet établissement étaient originaires des tribus indigènes de la région du Kouban, n’habitant le territoire de la péninsule de Taman que durant la saison des travaux des champs. Ainsi, l'établissement d'Artjuščenko-1 est le témoin d'un système spécifique d'intégration de la population barbare dans l'économie des colonies grecques de la région. Il est fort possible qu'en échange de leur travail, les Barbares aient reçu principalement du vin.

La découverte de graines brûlées montre que les agriculteurs, ici, plantaient surtout du blé tendre (Triticum aestivum s.l.) et de l'orge (Hordeum vulgare). Les légumineuses ne sont représentées qu'en relativement petite quantité. Ainsi, nous sommes ici en présence d'un bon exemple du «duo du Pont» mentionné ci-dessus. Je souligne encore une fois que le blé était la marchandise la plus importante dans l'exportation bosporane vers la Méditerranée, et sa culture présentait pour les colons grecs une signification toute particulière.

A propos des résultats de l'étude archéologique de l'établissement d'Artjuščenko-1 sur la péninsule de Taman, on retiendra que depuis le dernier tiers du VI $\mathrm{I}^{\mathrm{e}}$ siècle jusqu'au premier tiers $\mathrm{du} \mathrm{V} \mathrm{V}^{\mathrm{e}}$ s. av. J.-C. vivaient à cet endroit des travailleurs saisonniers, venus des territoires de la région du Kouban, peuplée par des tribus d'agriculteurs méotes. Le caractère saisonnier de la colonie peut s'expliquer par la situation démographique $\mathrm{du}$ Bosphore. En d'autres termes, ce sont à nouveau les déplacements des Scythes à travers le Bosphore Cimmérien qui ont pu influencer la formation d'un tel système d'utilisation des territoires agricoles.

Vers 480 av. J.-C., on observe un changement dans la situation militaire et politique du Bosphore. Ce changement fut probablement

54. Ju. A. Vinogradov, «Lepnaja keramika arkhaičeskogo vremeni s poselenija Artjuščenko-1 na Tamanskom poluostrove». 
Fig. 1 - Céramique modelée de type scythe provenant de l'établissement d'Artjuščenko-I. 
Fig. 2 - Céramique modelée de type méote provenant de l'établissement d'Artjuščenko-I. 
induit par une instabilité dans les steppes du nord de la mer Noire, suscitée par une agressivité croissante de la part des Scythes. Une telle situation peut s'expliquer par de nombreux facteurs, mais la progression vers l'est de nouvelles tribus scythes venues de l'ouest des steppes eurasiennes a dû revêtir, au final, une signification particulière. L'intensification de la tension militaire due à cette progression devint globale vers la fin du premier quart du $\mathrm{V}^{\mathrm{e}}$ s. av. J.-C. ${ }^{55}$. Le mobilier archéologique prouve que vers la même époque, certaines villes du Bosphore subirent des attaques, ce dont témoignent les couches d'incendies découvertes pendant les fouilles. Dans certaines d'entre elles, on a mis au jour de nouveaux édifices défensifs (Panticapée, Myrmèkion, Tiritaka, Porthmion, Phanagorie) ${ }^{56}$. Face à une telle situation, il semble que les poleis du Bosphore furent obligées de se lier dans une union défensive avec, à sa tête, la dynastie des Archéanactides (Diodore XII.31.1). Cette union a constitué un pas important sur la voie de la création de l'Etat monarchique du Bosphore.

Jurij Alekseevič Vinogradov

Institut d'Histoire de la Culture Matérielle, Saint-Pétersbourg

Traduction du russe de Mathilde Reichler IMPERIALI

55. K. K. Marčenko, Yu. A. Vinogradov, "The Scythian Period in the Northern Black Sea Region (750-250 BC)», p. 807 sq.; Ju. A. Vinogradov, "Cimmerian Bosporus", p. 146 et "Rhythms of Eurasia and the Main Historical Stages of the Kimmerian Bosporos in Pre-Roman Times», p. 15.

56. Cf. V. P. Tolstikov, «K probleme obrazovanija Bosporskogo gosudarstva", p. 24-48; Ju. A. Vinogradov, "Bospor Kimmerijskij", p. 238-245, 258-262; Ju. A. Vinogradov, V. A. Gorončarovskij, Voennaja istorija $i$ voennoe delo Bospora Kimmerijskogo (VI v. do n.è. - seredina III v. n.è.), p. 46-60. 


\section{BIBLIOGRAPHIE}

Abramov, Andrej Petrovič, Paromov, Jakov Maksimovič, «Ranneantičnye poselenija Tamanskogo poluostrova» ["Les établissements archaïques de la presqu'île de Taman»], Bosporskij sbornik, 2 (1993), p. 25-98.

Alekseev, Andrej Jur'evič, Khronografija evropejskoj Skifii VII-IV vv. do n.è. [Chronographie de la Scythie européenne des VII e-IVe siècles avant notre ère], Sankt-Peterburg, Izdatel'stvo Gosudarstvennogo Ermitaža, 2003.

Anoknin, Vladilen Afanas'evič, Istorija Bospora Kimmerijskogo [Histoire du Bosphore Cimmérien], Kiev, 1999.

Arsenyeva, Tatiana Mikhaylovna, "Tanais", in Ancient Greek Colonies in the Black Sea, ed. by Dimitrios V. Grammenos, Elias K. Petropoulos, Thessaloniki, Archaeological Institute of Northern Greece, 2003, vol. 2, p. 1047-1102.

Artamonov, Mikhail Illarionovič, Sokrovišča skifskikh kurganov v sobranii Gosudarstvennogo Ermitaža [Les trésors des kourganes scythes du Musée d'Etat de l'Ermitage], Praha/Leningrad, Artia, 1966.

Blavatskij, Vladimir Dmitrievič, «Arkhaičeskij Bospor» [«Le Bosphore archaïque»], $M I A, 33$ (1954), p. 7-44.

Brašinskij, Iosif Ben’jaminovič, Ščeglov, Aleksandr Nikolaevič, "Nekotorye problemy grečeskoj kolonizacii » ["Quelques questions sur la colonisation grecque»], in Problemy grečeskoj kolonizacii Severnogo i Vostočnogo Pričernomor'ja [Questions sur la colonisation grecque des littoraux nord et est de la mer Noire], otv. red. Otar Lordkipanidze, Tbilissi, Mecniereba, 1979, p. 29-46 (Materialy I Vsesojuznogo simpoziuma po drevnej istorii Pričernomor'ja: Ckhaltubo - 1977).

Braund, David, "Greater Olbia: Ethnic, Religious, Economic, and Political Interactions in the Region of Olbia, c. 600-100 BC», in Classical Olbia and the Scythian World. From the Sixth Century $B C$ to the Second Century AD, ed. by David Braund, S[ergej] 
D[mitrievič $]$ Kryzhitskiy, Oxford, Oxford University Press, 2007, p. 37-77.

Buyskikh, S[ergej] B[orisovičc], "Contacts between Greeks and NonGreeks on the Lower Bug in the Sixth and Fifth Centuries BC", in Classical Olbia and the Scythian World. From the Sixth Century $B C$ to the Second Century AD, ed. by David Braund, S[ergej] D[mitrievič] Kryzhitskiy, Oxford, Oxford University Press, 2007, p. 23-35.

Gajdukevič, Viktor Francevič, «Vinodelie na Bospore» [«L'industrie vinicole dans le Bosphore»], MIA, 85 (1958), p. 352-457.

—, Das Bosporanische Reich, Berlin, Akademie-Verlag, 1971.

Galanina, Ljudmila Konstantinovna, Grach, Nonna Leonidovna, Scythian Art. The legacy of the Scythian world: mid-7th to 3rd Century BC, Oxford/Leningrad, Phaidon/Aurora, 1987.

Graham, Alexander John, "Patterns in Early Greek Colonisation", Journal of Hellenic Studies, 91 (1971), p. 35-47.

Hansen, Mogens Herman, "The Hellenic Polis", in A comparative study of Thirty city-state cultures, ed. by Mogens Herman Hansen, Copenhagen, C. A. Reitzels Forlag, 2000, p. 141-187.

Inssen, Aleksandr Aleksandrovič, Grečeskaja kolonizacija Severnogo Pričernomor'ja, ejo predposylki i osobennosti [La colonisation grecque du littoral septentrional de la mer Noire, ses conditions et ses particularités], Leningrad, Gosudarstvennij Ermitaž, 1947.

Isager, Signe, Skydsgaard, Jens Erik, Ancient Greek Agriculture. An Introduction, London/New York, Routledge, 1992.

Kastanajan, Elizaveta Grigor'evna, Lepnaja keramika bosporskikh gorodov [La céramique modelée des villes bosporanes], Leningrad, Nauka, 1981.

Khazanov, Anatolij Mikhajlovič, Social'naja istorija Skifov [Histoire sociale des Scythes], Moskva, Nauka, 1975.

Kopejkina, Ljudmila Vladimirovna, «Raspisnaja rodossko-ionijskaja ojnokhoja iz kurgana Temir-Gora» "Une oenochoé rhodoionienne peinte provenant du kourgane de Temir-Gora»], Vestnik Drevnej Istorii, 1 (1972), p. 147-159.

Kopylov, Viktor Pavlovič, "Taganrogskoe poselenie v sisteme rannegrečeskikh kolonij Severnogo Pričernomor'ja” ["L'établissement de Taganrog dans le système des colonies 
grecques archaïques du littoral septentrional de la mer Noire»], Vestnik Drevnej Istorii, 4 (1999), p. 174-175.

Kopylov, Viktor Pavlovič, LarjonoK, Pavel Anatol'evič, Taganrogskoe poselenie [L'établissement de Taganrog], Rostov-na-Donu, Gefest, 1994.

Koshelenko, G[ennadij] A[ndreevič], Kuznetsov, V[ladimir] $\mathrm{D}$ [mitrievič], "Greek Colonisation of the Bosporus", in The Greek colonisation of the Black Sea area: historical interpretation of archaeology, ed. by Gocha R. Tsetskhladze, Stuttgart, Franz Steiner, 249-263.

Kruglikova, Irina Timofeevna, Sel'skoe khozjajstvo Bospora [L'économie agricole du Bosphore], Moskva, Nauka, 1976.

Kryžıtckij, Sergej Dmitrievič, Bujskikh, Sergej Borisovič, Burakov, Anatolij Vissarionovič, ОтrešKo, Valerij Mikhajlovič, Sel'skaja okruga Ol'vii [Le district agricole d'Olbia], Kiev, Naukova Dumka, 1989.

Kryžitckij, Sergej Dmitrievič, Bujskikh, Sergej Borisovič, OtrešKo, Valerij Mikhajlovič, Antičnye poselenija Nižnego Pobužja [Les établissements antiques du bas Boug], Kiev, Naukova Dumka, 1990.

Kryzhitskiy, S[ergej] D[mitrievič], "Criteria for the Presence of Barbarians in the Population of Early Olbia", in Classical Olbia and the Scythian World. From the Sixth Century BC to the Second Century AD, ed. by David Braund, S[ergej] D[mitrievič] Kryzhitskiy, Oxford, Oxford University Press, 2007, p. 17-22.

Kuznecov, Vladimir Dmitrievič, "Afiny i Bospor: khlebnaja torgovlja» ["Athènes et le Bosphore: le commerce céréalier»], Rossijskaja Arkheologija, 1 (2000), p. 107-120.

—, "Kepoi-Phanagoria-Taganrog", in Ancient Greek Colonies in the Black Sea, ed. by Dimitrios V. Grammenos, Elias K. Petropoulos, Thessaloniki, Archaeological Institute of Northern Greece, 2003, vol. 2, p. 895-955.

—, «Fanagorija: istorija issledovanija i novye nakhodki» [«Phanagorie: état de la recherche et nouvelles découvertes»], Rossijskaja Arkheologija, 2 (2007), p. 5-15.

Lapin, Vladimir Vasil'evič, Grečeskaja kolonizacija Severnogo Pričernomor' ja [La colonisation grecque du littoral septentrional de la mer Noire], Kiev, Naukova Dumka, 1966. 
MarČenko, Konstantin [Konstantinovič], Vinogradov, Yuri [Alekseevič], "The Scythian Period in the Northern Black Sea Region (750-250 BC)", Antiquity, 63 (1989), p. 803-813.

Maslennikov, Aleksandr Aleksandrovič, Ellinskaja khora na kraju Ojkumeny. Sel'skaja territorija evropejskogo Bospora v antičnuju èpokhu [La chôra hellénique en limite de l'oikouménè. Le territoire agricole de Bosphore européen à l'époque antique], Moskva, Indrik, 1998.

Minns, Ellis Hovell, Scythians and Greeks: a survey of ancient history and archaeology on the north coast of the Euxine from the Danube to the Caucasus, Cambridge, Cambridge University Press, 1913.

Murzin, Vyacheslav Yu[r'evič], Skory, Sergei A[natol'evič], "An Essay of Scythian History", Il Mar Nero, 1 (1994), p. 55-98.

Pashrevich, Galina A[leksandrovna], "Archaeobotanical studies on the northern coast of the Black Sea", Eurasia Antiqua, 7 (2001), p. 511-567.

Rostovtzeff, M[ikhail Ivanovič], Iranians and Greeks in South Russia, Oxford, Clarendon Press, 1922.

SAPrykin, Sergej Ju[r'evič], "The Chora in the Bosporan Kingdom", in Surveying the Greek Chora: Black Sea region in a comparative perspective, ed. by Pia Guldager Bilde, Vladimir Stolba, Aarhus, Aarhus University Press, 2006, p. 273-288.

Sarpaki, Anaya, "The Palaeoethnobotanical Approach. The Mediterranean Triad or Is It a Quartet?", in Agriculture in Ancient Greece. Proceedings of the Seventh International Symposium at the Sweden Institute at Athens, ed. by Berit Wells, Stokholm, P. Åström, 1992, p. 61-76.

Šelov, Dmitrij Borisovič, Tanais i Nižnij Don v III-I vv. do n.è. [Tanaïs et le bas Don entre le III et le I ${ }^{e r}$ siècle avant notre ère], Moskva, Nauka, 1970.

-, Coinage of the Bosporus VI-II centuries BC, Oxford, Archaeopress, 1978 (BAR International Series 46).

— «Tanais - èllinističeskij gorod» ["Tanaïs - une ville hellénistique»], Vestnik Drevnej Istorii, 3 (1989), p. 47-54.

Šelov-Kovedjaev, Fjodor Vadimovič, «Istorija Bospora v VI-IV vv. do n. è.» ["Histoire du Bosphore aux VI ${ }^{\mathrm{e}}-\mathrm{IV}^{\mathrm{e}}$ siècles avant notre ère"], in Drevnejšie gosudarstva na territorii SSSR. Materialy 
i issledovanija 1984 [Les Etats les plus anciens sur le territoire de l'URSS], Moskva, 1985, p. 5-187.

TerešČenko, A[ndrej] E[vgen'evič], "O pojavlenii monetnogo dela na Bospore Kimmerijskom» "Sur l'apparition de la monnaie dans le Bosphore Cimmérien»], in Bosporskij fenomen: Pogrebal'nye pamjatniki i svjatilišča. Materialy meždunarodnoj naučnoj konferencii [Le phénomène bosporan: monuments funéraires et sanctuaires. Actes du colloque international], otv. red. Marina Jur'evna Vakhtina, Vadim Jur'evič Zuev, Sergej Vladimirovič Kašaev, Vladimir Andreevič Khršanovskij, Sankt-Peterburg, 2002, vol. 1, p. 206-209.

Tolstikov, Vladimir Petrovič, «K probleme obrazovanija Bosporskogo gosudarstva (opyt rekonstrukcii voenno-političeskoj situacii na Bospore v konce VI - pervoj polovine V v. do n. è.) " ["La question de la formation de l'Etat bosporan (essai de reconstitution de la situation militaro-politique au Bosphore à la fin du VIe siècle et durant la première moitié $\mathrm{du} \mathrm{V}^{\mathrm{e}}$ siècle avant notre ère)»], Vestnik Drevnej Istorii, 3 (1984), p. 24-48.

Tsetsknladze, Gocha R., "A Survey of the Major Urban Settlements in the Cimmerian Bosporos (With a Discussion of their Status as Poleis)", in Yet More Studies in the Ancient Greek Polis, ed. by Thomas Heine Nielsen, Stuttgart, Franz Steiner, 1997, p. 39-81.

—, "On the Earliest Greek Colonial Architecture in the Pontus", in Pontus and the outside world: studies in Black Sea history, historiography, and archaeology, ed. by Christopher J. Tuplin, Leiden/ Boston, Brill, 2004, p. 225-278.

Vakhtina, Marina Jur'evna, «Porfmij - grečeskij gorod u perepravy čerez Kimmerijskij Bospor» ["Porthmion - une ville grecque au passage du Bosphore Cimmérien»], Bosporskie issledovanija, 22 (2009), p. 91-108.

Vakhtina, Marina Jur'evna, Vinogradov, Jurij Alekseevič, Rogov, Evgenij Jakovlevič, "Ob odnom iz maršrutov voennykh pokhodov i sezonnykh migracij kočevykh Skifov» " A propos de l'un des trajets empruntés par les Scythes nomades lors de leurs campagnes militaires et de leurs migrations saisonnières»], Vestnik Drevnej Istorii, 4 (1980), p. 155-161.

Vakhtina, Marina Jur'evna, Vinogradov, Jurij Alekseevič, «Eščje raz o rannej fortifikacii Bospora Kimmerijskogo» ["Retour sur la 
question de la fortification ancienne du Bosphore Cimmérien»], in Bosporskij fenomen: kolonizacija regiona, formirovanie polisov, obrazovanie gosudarstva. Materialy meždunarodnoj naučnoj konferencii [Le phénomène bosporan: colonisation de la région, formation des poleis et de l'Etat. Actes de colloque international], otv. red. Marina Jur'evna Vakhtina, Vadim Jur'evič Zuev, Evgenij Jakovlevič Rogov, Vladimir Andreevič Khršanovskij, SanktPeterburg, 2001, vol. 1, p. 41-45.

Vinogradov, Jurij Alekseevič, «Mirmekij» ["Myrmèkion»], in Očerki arkheologii $i$ istorii Bospora [Essais d'archéologie et d'histoire du Bosphore], otv. red. Gennadij Andreevič Košelenko et al., Moskva, Nauka, 1992, p. 99-120.

—, «K probleme polisov v rajone Bospora Kimmerijskogo » « «a question des poleis dans la région du Bosphore Cimmérien»], Antičnij mir i arkheologija, 9 (1993), p. 79-96.

—, «Nekotorye diskussionnye problemy grečeskoj kolonizacii Bospora Kimmerijskogo" [Quelques questions sujettes à discussion autour de la colonisation grecque du Bosphore Cimmérien »], Vestnik Drevnej Istorii, 3 (1995), p. 152-160.

—, «Prirodnij faktor v razvitii Bospora v skifskuju èpokhu (Nekotorye aspekty izučenija)» " Le facteur naturel dans le développement du Bosphore à l'époque scythe. (Quelques aspects de cette étude)»], Vestnik Drevnej Istorii, 3 (1996), p. 77-88.

-, "Selecting Findings from the Myrmekion Acropolis", Etudes et travaux, 18 (1999), p. 280-293.

—, «Grečeskaja kolonizacija i grečeskaja urbanizacija Severnogo Pričernomor' ja» [ "La colonisation et l'urbanisation grecques du littoral septentrional de la mer Noire»], Stratum plus, 3 (1999), p. $101-115$.

—, «K probleme stanovlenija drevnegrečeskikh gorodov v rajone Bospora Kimmerijskogo" ["Problème de la formation des villes grecques anciennes dans la région du Bosphore Cimmérien»], in $\Sigma \Upsilon \Sigma \Sigma$ ITAI. Pamjati Jurija Viktoroviča Andreeva [ $\Sigma \Upsilon \Sigma \Sigma$ ITAI. En mémoire de Jurij Viktorovič Andreev], otv. red. Vadim Jur'evič Zuev, Sankt-Peterburg, Aletejja, 2000, p. 227-232.

—, "Bospor Kimmerijskij» ["Le Bosphore Cimmérien»], in Greki i Varvary Severnogo Pričernomor'ja v skifskuju èpokhu [Les Grecs et les Barbares du littoral septentrional de la mer Noire à l'époque 
scythe], otv. red. Konstantin Konstantinovič Marčenko, SanktPeterburg, Aletejja, 2005, p. 211-296.

—, "K izučeniju zeren kul'turnykh rastenij, najdennykh v Mirmekii » ["A propos de l'étude des graines de plantes cultivées, trouvées à Myrmèkion"], Khersonesskij sbornik, 14 (2005), p. 94-98.

—, "Lepnaja keramika arkhaičeskogo vremeni s poselenija Artjuščenko-1 na Tamanskom poluostrove» [La céramique modelée de l'époque archaïque en provenance de l'établissement d'Artjuščenko-1 sur la presqu'île de Taman »], Zapiski Instituta istorii material'noj kul'tury RAN, 1 (2006), p. 69-76.

—, "Cimmerian Bosporus: Main Phases of Pre-Roman History", in Greeks and Natives in the Cimmerian Bosporus, 7th-1st Centuries $B C$ : proceedings of the international conference October 2000, ed. by Sergej L['vovič] Solovyov, Taman, 2007, p. 145-149 (BAR International Series 1729).

—, "Nekotorye itogi arkheologičeskikh issledovanij v rajone akropolja Mirmekija (Po materialam raskopok 1992-1994 gg)» ["Quelques résultats des investigations archéologiques menées dans la région de l'acropole de Myrmèkion (d'après le mobilier des fouilles de 1992 à 1994) »], Vestnik Drevnej Istorii, 1 (2008), p. 49-52.

-, "Rhythms of Eurasia and the Main Historical Stages of the

Kimmerian Bosporos in Pre-Roman Times", in Meetings of Cultures in the Black Sea Region. Between Conflict and Coexistence, ed. by Pia Guldager Bilde, Jane Hjarl Petersen, Aarhus, Aarhus University Press, 2008, p. 13-27 (Black Sea Studies 8).

—, "Die Befestung der Akropolis von Myrmekion", in Die Griechen und ihre Nachbarn am Nordland des Schwarzen Meeres. Internationales archäologisches Kolloquium Münster 2001, hrsg. von Klaus Stähler, Gabriele Gudrian, Münster, Ugarit Verlag, 2009, p. 95-114 (Eikon 9).

—, "The Cimmerian Bosporus: the Main Stages of History", in Le grec du monde colonial antique. I. Le Nord et Nord-Ouest de la mer Noire. Actes de la table ronde de Nancy 28-29 septembre 2007, éd. par Guy Vottéro, Nancy, 2009, vol. 1, p. 7-32 (Etudes anciennes 42).

Vinogradov, Jurij Alekseevič, Butyagin, Alexandr Mikhaylovitch, Vakhtina, Marina Yuryevna, "Myrmekion - Porthmeus. Two "small" towns of the Ancient Bosporus", in Ancient Greek 
Colonies in the Black Sea, ed. by Dimitrios V. Grammenos, Elias K. Petropoulos, Thessaloniki, 2003, vol. 2, p. 803-840.

Vinogradov, Jurij Alekseevič, Lebedeva, Elena Vladimirovna, "Excavations at the Classical-Period Settlement of Artyushchenko-1 (Bugazskyoe) on the Taman Peninsula", Hyperboreus, 11/2 (2005), p. 316-319.

Vinogradov, Jurij Alekseevič, GorončarovskiJ, Vladimir Anatol'evič, Voennaja istorija i voennoe delo Bospora Kimmerijskogo (VI v. do n.è. - seredina III v. n.è.) [Histoire militaire et militaria du Bosphore Cimmérien (VIe siècle avant notre ère - moitié du III siècle de notre ère], Sankt-Peterburg, Nestor-Istorija, 2009.

Vinogradov, Jurij Germanovič, Pontische Studien. Kleine Schriften zur Geschichte und Epigraphik des Schwarzmeerraumes, Mainz, Philipp von Zabern, 1997.

\section{Crédits iconographiques}

Fig. 1, 2, pl. III.3:

Dessins de Ju. A. Vinogradov.

Pl. II :

Infographie de Laurent Saget (C) UNIL/IASA.

Pl. III.1, IV.3 et V:

Photos de Ju. A. Vinogradov.

Pl. III.2 et IV.1-2:

Photos et dessin de S. V. Jabločkin. 
